\title{
The crystal structure of $\mathrm{N}$-(1-(dimethyl-14-azanylidene)ethyl)propan-2- amine, a $Z^{\prime}>1$ structure, $\mathrm{C}_{8} \mathrm{H}_{18} \mathrm{~N}_{2}$
}

\author{
Gamall Makhloufi, Kai Schütte and Christoph Janiak ${ }^{*}$
}

Institut für Anorganische Chemie und Strukturchemie, Lehrstuhl I: Bioanorganische Chemie und Katalyse, Heinrich-Heine-Universität Düsseldorf, Universitätsstrasse 1, D-40225 Düsseldorf, Germany

Received September 08, 2014, accepted October 30, 2014, available online November 07, 2014, CCDC no. 1267/4223

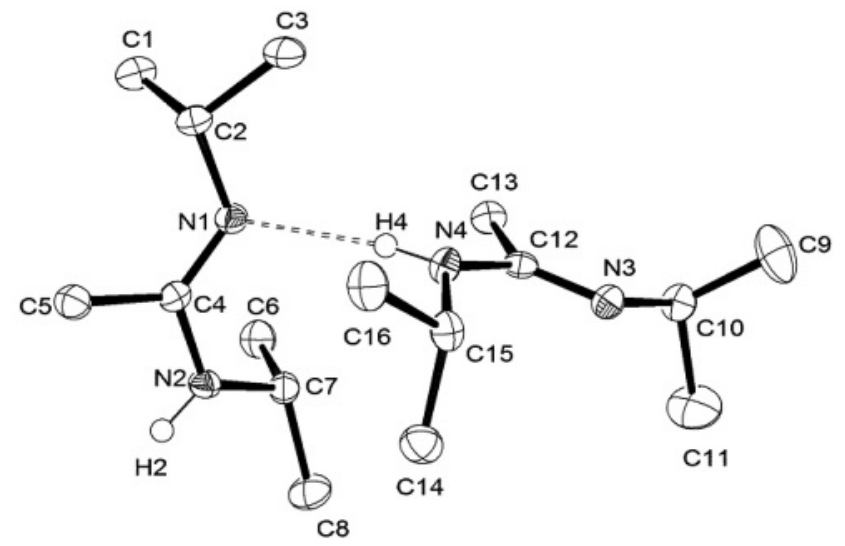

\section{Abstract}

$\mathrm{C}_{8} \mathrm{H}_{18} \mathrm{~N}_{2}$, monoclinic, $P 2{ }_{1} / n$ (no. 14), $a=9.708(9) \AA$,

$b=14.988(17) \AA, c=13.207(12) \AA, \beta=95.92(3)^{\circ}$,

$V=1911.4 \AA^{3}, Z=8, R_{\mathrm{gt}}(F)=0.0369, w R_{\mathrm{ref}}\left(F^{2}\right)=0.0937$,

$T=100 \mathrm{~K}$

Table 1. Data collection and handling.

Crystal:

Wavelength:

$\mu$ :

Diffractometer, scan mode:

$2 \theta_{\max }:$

$N(h k l)_{\text {measured }}, N(h k l)_{\text {unique }}$ :

Criterion for $I_{\mathrm{obs}}, N(h k l)_{\mathrm{gt}}$ :

$N(\text { param })_{\text {refined }}$

Programs:

\section{Source of material}

The synthesis was carried out with standard Schlenk techniques under nitrogen atmosphere since the lithium amidinate is hygroscopic and air sensitive. The solvents ( $n$-hexane and tetrahydrofuran) were dried using the MBraun solvent purification system. $N$-(1-(dimethyl-14-azanylidene)ethyl)propan-2amine was synthesized by deprotonation and methylation of 1,3diisopropylcarbo- diimide with methyllithium according to literature procedures $[2,3]$. The resulting lithium amidinate was then reacted with a stoichiometric amount of water and purified by sublimation to afford colourless crystals $\left(800 \mathrm{mbar}, 25^{\circ} \mathrm{C}\right)$.

\section{Experimental details}

The two N-bound hydrogen atoms were found in difference Fourier syntheses and were refined freely $(\mathrm{H} 2, \mathrm{H} 4)$. The hydrogen atoms of the isopropyl groups were idealized and refined using rigid groups. For the $\mathrm{CH}_{3}$-groups AFIX 137 (with $U_{\text {iso }}(\mathrm{H})=$

\footnotetext{
* Correspondence author (e-mail: janiak@hhu.de)
}

$\left.1.5 U_{\text {eq }}(\mathrm{C})\right)$ and for the CH-group AFIX 13 option $\left(U_{\text {iso }}(\mathrm{H})=\right.$ $\left.1.2 U_{\mathrm{eq}}(\mathrm{C})\right)$ of the SHELXL-2013 program was used [1].

\section{Discussion}

Metal amidinates are investigated and widely used as precursors for thin metal films in low pressure chemical vapor deposition (CVD) or atomic layer deposition (ALD) [2-5]. They also can be used as precursors for mono- and bimetallic nanoparticle synthesis [6]. Here we show that hydrolytic decomposition of metal amidinates like $\left[\mathrm{Me}\left(\mathrm{C}\left(\mathrm{N}^{\mathrm{i}} \mathrm{Pr}\right)_{2}\right)\right]_{2} \mathrm{Zn}$ or $\left\{\left[\mathrm{Me}\left(\mathrm{C}\left(\mathrm{N}^{\mathrm{i}} \mathrm{Pr}\right)_{2}\right)\right] \mathrm{Li}\right\}_{2}$ yields the free $\mathrm{N}$-(1-(dimethyl-14-azanylidene)ethyl)propan-2amine molecule. Two crystallographically independent mole-

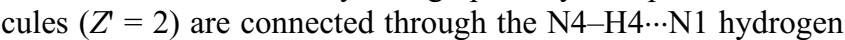
bond to form the asymmetric unit ( $c f$. Fig.). $Z$ is the number of formula units in the unit cell divided by the number of independent general positions $[7,8]$. There is an ongoing discussion on the origin of so-called $Z^{\prime}>1$ structures. Different ideas have been put forward to rationalize this phenomenon [9]: A "fossil relic" of a more stable form [10], a crystal "on the way" $[11,12]$ or strong and special supramolecular interactions between the two (or more) symmetry-independent units [13-15]. A high $Z^{\prime}$ is also obtained when the molecule has different equi-energetic conformations, with these conformations co-existing in the crystal [16]. An overlay of the two symmetry-independent molecules of the title structure shows a small difference in $\mathrm{C}-\mathrm{N}-\mathrm{C}\left({ }^{\mathrm{i}} \mathrm{Pr}\right)-\mathrm{C}\left({ }^{\mathrm{i}} \mathrm{Pr}\right)$ torsion angles. Hence, we trace the co-existence of two crystallographically independent molecules to the presence of the hydrogen bond. The di-molecular unit is part of a supramolecular hydrogen-bridged chain structure which extends along the $a c$-diagonal. Neighboring chains are arranged in parallel through van der Waals contacts.

Table 2. Atomic coordinates and displacement parameters (in $\AA^{2}$ ).

\begin{tabular}{llllll}
\hline Atom & Site & $x$ & $y$ & $z$ & $U_{\text {iso }}$ \\
\hline $\mathrm{H}(1 \mathrm{~A})$ & $4 e$ & 0.2072 & 0.5430 & 1.0191 & 0.041 \\
$\mathrm{H}(1 \mathrm{~B})$ & $4 e$ & 0.0957 & 0.5935 & 1.0795 & 0.041 \\
$\mathrm{H}(1 \mathrm{C})$ & $4 e$ & 0.2530 & 0.6271 & 1.0893 & 0.041 \\
$\mathrm{H}(2 \mathrm{~A})$ & $4 e$ & 0.1159 & 0.7232 & 0.9767 & 0.025 \\
$\mathrm{H}(3 \mathrm{~A})$ & $4 e$ & 0.0016 & 0.6649 & 0.8235 & 0.046 \\
$\mathrm{H}(3 \mathrm{~B})$ & $4 e$ & -0.0562 & 0.6156 & 0.9179 & 0.046 \\
$\mathrm{H}(3 \mathrm{C})$ & $4 e$ & 0.0535 & 0.5664 & 0.8546 & 0.046 \\
$\mathrm{H}(5 \mathrm{~A})$ & $4 e$ & 0.2586 & 0.8412 & 0.9857 & 0.034 \\
$\mathrm{H}(5 \mathrm{~B})$ & $4 e$ & 0.4234 & 0.8447 & 1.0104 & 0.034 \\
$\mathrm{H}(5 \mathrm{C})$ & $4 e$ & 0.3342 & 0.7769 & 1.0708 & 0.034 \\
$\mathrm{H}(2)$ & $4 e$ & $0.540(2)$ & $0.777(1)$ & $0.907(1)$ & $0.030(4)$ \\
$\mathrm{H}(6 \mathrm{~A})$ & $4 e$ & 0.6502 & 0.6003 & 0.9242 & 0.041 \\
$\mathrm{H}(6 \mathrm{~B})$ & $4 e$ & 0.5917 & 0.5390 & 0.8303 & 0.041 \\
$\mathrm{H}(6 \mathrm{C})$ & $4 e$ & 0.4927 & 0.5672 & 0.9145 & 0.041 \\
$\mathrm{H}(7)$ & $4 e$ & 0.4467 & 0.6539 & 0.7627 & 0.024 \\
$\mathrm{H}(8 \mathrm{~A})$ & $4 e$ & 0.6116 & 0.7576 & 0.7173 & 0.046 \\
\hline
\end{tabular}


Table 2. continued.

\begin{tabular}{llllll}
\hline Atom & Site & $x$ & $y$ & $z$ & $U_{\text {iso }}$ \\
\hline $\mathrm{H}(8 \mathrm{~B})$ & $4 e$ & 0.6748 & 0.6591 & 0.7145 & 0.046 \\
$\mathrm{H}(8 \mathrm{C})$ & $4 e$ & 0.7207 & 0.7238 & 0.8083 & 0.046 \\
$\mathrm{H}(9 \mathrm{~A})$ & $4 e$ & 0.1101 & 0.5857 & 0.3154 & 0.063 \\
$\mathrm{H}(9 \mathrm{~B})$ & $4 e$ & 0.0489 & 0.5159 & 0.3908 & 0.063 \\
$\mathrm{H}(9 \mathrm{C})$ & $4 e$ & 0.1609 & 0.4840 & 0.3176 & 0.063 \\
$\mathrm{H}(10)$ & $4 e$ & 0.2677 & 0.5031 & 0.4854 & 0.027 \\
$\mathrm{H}(11 \mathrm{~A})$ & $4 e$ & 0.4527 & 0.5949 & 0.4495 & 0.069 \\
$\mathrm{H}(11 \mathrm{~B})$ & $4 e$ & 0.3609 & 0.6379 & 0.3543 & 0.069 \\
$\mathrm{H}(11 \mathrm{C})$ & $4 e$ & 0.4066 & 0.5354 & 0.3512 & 0.069 \\
$\mathrm{H}(13 \mathrm{~A})$ & $4 e$ & 0.3526 & 0.5200 & 0.6491 & 0.033 \\
\hline
\end{tabular}

Table 2. continued.

\begin{tabular}{llllll}
\hline Atom & Site & $x$ & $y$ & $z$ & $U_{\text {iso }}$ \\
\hline $\mathrm{H}(13 \mathrm{~B})$ & $4 e$ & 0.1951 & 0.4868 & 0.6362 & 0.033 \\
$\mathrm{H}(13 \mathrm{C})$ & $4 e$ & 0.2515 & 0.5452 & 0.7331 & 0.033 \\
$\mathrm{H}(4)$ & $4 e$ & $0.200(2)$ & $0.681(1)$ & $0.730(1)$ & $0.038(4)$ \\
$\mathrm{H}(14 \mathrm{~A})$ & $4 e$ & 0.3649 & 0.7903 & 0.5793 & 0.044 \\
$\mathrm{H}(14 \mathrm{~B})$ & $4 e$ & 0.3634 & 0.8261 & 0.6934 & 0.044 \\
$\mathrm{H}(14 \mathrm{C})$ & $4 e$ & 0.3013 & 0.8864 & 0.5996 & 0.044 \\
$\mathrm{H}(15)$ & $4 e$ & 0.1190 & 0.7841 & 0.5609 & 0.026 \\
$\mathrm{H}(16 \mathrm{~A})$ & $4 e$ & 0.1336 & 0.8317 & 0.7719 & 0.044 \\
$\mathrm{H}(16 \mathrm{~B})$ & $4 e$ & -0.0059 & 0.8077 & 0.7018 & 0.044 \\
$\mathrm{H}(16 \mathrm{C})$ & $4 e$ & 0.0776 & 0.8967 & 0.6807 & 0.044 \\
\end{tabular}

Table 3. Atomic coordinates and displacement parameters (in $\AA^{2}$ ).

\begin{tabular}{|c|c|c|c|c|c|c|c|c|c|c|}
\hline Atom & Site & $x$ & $y$ & $z$ & $U_{11}$ & $U_{22}$ & $U_{33}$ & $U_{12}$ & $U_{13}$ & $U_{23}$ \\
\hline $\mathrm{C}(1)$ & $4 e$ & $0.1780(1)$ & $0.60113(9)$ & $1.0432(1)$ & $0.0245(6)$ & $0.0293(8)$ & $0.0287(7)$ & $-0.0009(5)$ & $0.0069(6)$ & $0.0046(6)$ \\
\hline$C(2)$ & $4 e$ & $0.1438(1)$ & $0.66340(9)$ & $0.95202(9)$ & $0.0175(6)$ & $0.0221(7)$ & $0.0227(7)$ & $0.0000(5)$ & $0.0062(5)$ & $0.0014(5)$ \\
\hline$C(3)$ & $4 e$ & $0.0250(1)$ & $0.6241(1)$ & $0.8806(1)$ & $0.0192(6)$ & $0.0426(9)$ & $0.0307(8)$ & $-0.0079(6)$ & $0.0056(6)$ & $0.0013(6)$ \\
\hline $\mathrm{N}(1)$ & $4 e$ & $0.2671(1)$ & $0.67322(7)$ & $0.89644(8)$ & $0.0154(5)$ & $0.0232(6)$ & $0.0196(6)$ & $-0.0003(4)$ & $0.0032(4)$ & $0.0000(4)$ \\
\hline $\mathrm{C}(4)$ & $4 e$ & $0.3569(1)$ & $0.73488(8)$ & $0.92476(9)$ & $0.0157(6)$ & $0.0189(7)$ & $0.0166(6)$ & $0.0027(5)$ & $0.0016(5)$ & $0.0039(5)$ \\
\hline$C(5)$ & $4 e$ & $0.3419(1)$ & $0.80569(8)$ & $1.0051(1)$ & $0.0210(6)$ & $0.0213(7)$ & $0.0273(7)$ & $0.0000(5)$ & $0.0054(6)$ & $-0.0023(6)$ \\
\hline$C(6)$ & $4 e$ & $0.5689(1)$ & $0.58669(9)$ & $0.8765(1)$ & $0.0291(7)$ & $0.0237(7)$ & $0.0288(7)$ & $0.0036(6)$ & $0.0038(6)$ & $-0.0026(6)$ \\
\hline$C(7)$ & $4 e$ & $0.5249(1)$ & $0.67039(8)$ & $0.81458(9)$ & $0.0166(6)$ & $0.0250(7)$ & $0.0190(6)$ & $0.0000(5)$ & $0.0022(5)$ & $-0.0022(5)$ \\
\hline $\mathrm{C}(8)$ & $4 e$ & $0.6436(1)$ & $0.7059(1)$ & $0.7587(1)$ & $0.0256(7)$ & $0.0332(8)$ & $0.0359(8)$ & $0.0025(6)$ & $0.0139(6)$ & $0.0000(6)$ \\
\hline $\mathrm{C}(9)$ & $4 e$ & $0.1315(2)$ & $0.5335(1)$ & $0.3589(1)$ & $0.0387(8)$ & $0.0386(9)$ & $0.0453(9)$ & $0.0105(7)$ & $-0.0089(8)$ & $-0.0219(7)$ \\
\hline$C(10)$ & $4 e$ & $0.2472(1)$ & $0.55655(9)$ & $0.4410(1)$ & $0.0233(6)$ & $0.0214(7)$ & $0.0240(7)$ & $0.0054(5)$ & $0.0046(6)$ & $-0.0023(5)$ \\
\hline $\mathrm{C}(11)$ & $4 e$ & $0.3785(2)$ & $0.5836(1)$ & $0.3949(1)$ & $0.0364(8)$ & $0.046(1)$ & $0.060(1)$ & $0.0027(7)$ & $0.0261(8)$ & $-0.0143(8)$ \\
\hline$N(3)$ & $4 e$ & $0.2052(1)$ & $0.63099(7)$ & $0.50410(8)$ & $0.0193(5)$ & $0.0198(6)$ & $0.0200(6)$ & $0.0031(4)$ & $0.0047(5)$ & $-0.0017(4)$ \\
\hline $\mathrm{C}(12)$ & $4 e$ & $0.2146(1)$ & $0.62066(8)$ & $0.60188(9)$ & $0.0103(5)$ & $0.0198(7)$ & $0.0234(7)$ & $0.0000(5)$ & $0.0037(5)$ & $0.0006(5)$ \\
\hline $\mathrm{N}(4)$ & $4 e$ & $0.1831(1)$ & $0.68941(7)$ & $0.66430(9)$ & $0.0224(5)$ & $0.0195(6)$ & $0.0171(6)$ & $0.0034(4)$ & $0.0024(5)$ & $0.0005(4)$ \\
\hline$C(14)$ & $4 e$ & $0.3128(1)$ & $0.82520(9)$ & $0.6252(1)$ & $0.0311(7)$ & $0.0245(7)$ & $0.0311(8)$ & $-0.0024(6)$ & $0.0011(6)$ & $0.0041(6)$ \\
\hline$C(15)$ & $4 e$ & $0.1704(1)$ & $0.78279(8)$ & $0.6305(1)$ & $0.0243(6)$ & $0.0194(7)$ & $0.0198(7)$ & $0.0038(5)$ & $-0.0018(6)$ & $-0.0001(5)$ \\
\hline$C(16)$ & $4 e$ & $0.0864(2)$ & $0.83431(9)$ & $0.7027(1)$ & $0.0341(7)$ & $0.0238(7)$ & $0.0297(8)$ & $0.0072(6)$ & $0.0011(6)$ & $-0.0044(6)$ \\
\hline
\end{tabular}

Acknowledgments. I acknowledge support for the publication fee by the Deutsche Forschungsgemeinschaft (DFG) and the open access publication fund of the Heinrich-Heine-Universität Düsseldorf.

\section{References}

1. Sheldrick, G. M.: A short history of SHELX. Acta Crystallogr. A64( 2008) 112-122.

2. Lim, B.S.; Rahtu, A.; Park, J.-S.; Gordon, R. G.: Synthesis and Characterization of Volatile, Thermally Stable, Reactive Transition Metal Amidinates. Inorg. Chem. 42 (2003) 7951-7958.

3. Schmidt, S.; Schulz, S.; Blaeser, D.; Boese, R.; Bolte, M.: Synthesis and Structural Characterization of New Zinc Amidinate Complexes. Organometallics 29 (2010) 6097-6103.

4. Krisyuk, V.; Aloui, L.; Prud'homme, N.; Sysoev, S.; Senocq, F.; Samelor, D.; Vahlas, C.: CVD of Pure Copper Films from Amidinate Precursor Electrochemical/Chemical Deposition and Etching. Electrochem. Solid State Lett. 14 (2011) D26-D29.

5. Bahlawane, N.; Kohse-Hoinghaus, K.; Premkumar, P. A.; Lenoble, D.: Advances in the deposition chemistry of metal-containing thin films using gas phase processes. Chem. Sci. 3 (2012) 929-941.

6. Schütte, K.; Meyer, H.; Gemel, C.; Barthel, J.; Fischer, R. A.; Janiak, C.: Synthesis of $\mathrm{Cu}, \mathrm{Zn}$ and $\mathrm{Cu} / \mathrm{Zn}$ brass alloy nanoparticles from metal amidinate precursors in ionic liquids or propylene carbonate with relevance to methanol synthesis. Nanoscale 6 (2014) 3116-3126.

7. Ruiz, J.; Rodriuez, V.; Cutillas, N.; Hoffmann, A.; Chamayou, A.-C.; Kazmierczak; K.; Janiak, C.: Structure-solid-state CPMAS ${ }^{13}$ C NMR correlation in palladacycle solvates (pseudo-polymorphs) with a transformation from $Z^{\prime}=1$ to $Z^{\prime}=2$. CrystEngComm. 10 (2008) 1928-1938.
8. Chamayou, A.-C.; Biswas, C.; Ghosh, A.; Janiak, C.: (Acetato$\kappa O)$ aqua $\left(1 H\right.$-imidazole- $\left.\mathrm{N}^{3}\right)\left(\right.$ picolinato- $\left.\kappa^{2} N, O\right)$ copper(II) 0.87 -hydrate: a $Z^{\prime}>1$ structure. Acta Cryst. C65 (2009) m311-m313.

9. Gavezotti, A.: Structure and energy in organic crystals with two molecules in the asymmetric unit: causality or chance?. CrystEngComm. 10 (2008) 389-398.

10. Nichol, G. S.; Clegg, W.: Further thoughts on crystal structures with Z' > 1: analysis of single-crystal structures determined using X-ray synchrotron and neutron radiation in the Cambridge Structural Database. CrystEngComm. 9 (2007) 959-960.

11. Steed, J. W.: Should solid-state molecular packing have to obey the rules of crystallographic symmetry?. CrystEngComm. 5 (2003) 169-179.

12. Desiraju, G. R.: On the presence of multiple molecules in the crystal asymmetric unit $\left(Z^{\prime}>1\right)$. CrystEngComm. 9 (2007) 91-92.

13. Althoff, G.; Ruiz, J.; Rodríguez, V.; López, G.; Pérez, J.; Janiak, C.: Can a single $\mathrm{C}-\mathrm{H} \cdots \mathrm{F}-\mathrm{C}$ hydrogen bond make a difference? Assessing the $\mathrm{H} \cdots \mathrm{F}$ bond strength from 2-D ${ }^{1} \mathrm{H}-{ }^{19} \mathrm{~F}$ CP/MAS NMR. CrystEngComm. 8 (2006) 662-665.

14. Hao, X.; Parkin, S.; Brock, C. P.: The unusual phases of anhydrous and hydrated pinacol. Acta Crystallogr. B61 (2005) 689-699.

15. Babu, N. J.; Nangia, A.: High $Z^{\prime}$ polymorphs have shorter $\mathrm{C}-\mathrm{H} \cdots \mathrm{O}$ interactions and $\mathrm{O}-\mathrm{H} \cdots \mathrm{O}$ hydrogen bonds. CrystEngComm. 9 (2007) 980-983.

16. Roy, S.; Banerjee, R.; Nangia, A.; Kruger, G. J.: Conformational, Concomitant Polymorphs of 4,4-Diphenyl-2,5-cyclohexadienone: Conformation and Lattice Energy Compensation in the Kinetic and Thermodynamic Forms. Chem. Eur. J. 12 (2006) 3777-3788. 